\title{
AREA MINIMIZERS IN A K3 SURFACE AND HOLOMORPHICITY
}

\author{
M. MICALLEF AND J. WOLFSON
}

\section{Introduction}

The following is a well known consequence of the Wirtinger inequality: a compact complex submanifold of a Kähler manifold is a volume minimizer in its homology class and any other volume minimizer in that class is, necessarily, complex. In particular, in a Kähler surface a holomorphic curve is an area minimizer in its homology class. In light of this result it is natural, given a Kähler surface, to investigate the relation between area minimizers and complex curves. When the ambient manifold is a flat fourdimensional torus it was shown in $[\mathrm{M}]$, using second variation arguments, that a two-dimensional area minimizer is holomorphic for one of the complex structures compatible with the metric. In [MW] the authors attempted to prove an analogous result for a Ricci flat (Calabi-Yau) metric $g$ on a K3 surface $X$. Such a metric is hyperkähler in the sense that there is a two-sphere of complex structures, called the hyperkähler line, each of which is compatible with $g$. We obtained partial positive results which are extended in section 5 of this paper. We also showed that there is a strictly stable minimal two-sphere in a (non-compact) hyperkähler surface that is not holomorphic for any compatible complex structure. This shows that second variation arguments cannot be used to answer this question.

However there is compelling evidence for a result on K3 surfaces analogous to that for the flat torus. It can be shown that if a cohomology class $\alpha \in H^{1,1}(X ; \mathbb{R}) \cap H^{2}(X ; \mathbb{Z})$ satisfies $\alpha \cdot \alpha \geq-2$ then its Poincaré dual can be represented by a curve that is holomorphic. Moreover, there is a set of generators of $H_{2}(X ; \mathbb{Z})$ each of which can be represented by a curve holomorphic for some complex structure on the hyperkähler line. Thus every class $\gamma \in H_{2}(X ; \mathbb{Z})$ can be represented by a sum of curves each of which is holomorphic for some complex structure on the hyperkähler line. A minimizer of area among surfaces representing $\gamma$ consists of a sum of branched immersed surfaces

$$
\Sigma_{1} \cup \cdots \cup \Sigma_{k}
$$

and it is then reasonable to ask whether each $\Sigma_{i}$ is holomorphic for some complex structure on the hyperkähler line determined by $g$. Though this

Date: February 1, 2008.

The second author was partially supported by NSF grant DMS-0304587. 
is true for many homology classes we show, in this paper, that there is an integral homology class $\gamma$ and a hyperkähler metric $g$ such that no area minimizer of $\gamma$ has this property. Thus the result for flat four-tori does not carry over to K3 surfaces. For recent work on similar problems in KählerEinstein manifolds see [AN].

For lagrangian area minimizers and lagrangian homology classes there are analogous questions. Given a lagrangian homology class $\gamma \in H_{2}(X ; \mathbb{Z})$ a minimizer of area among lagrangian two-spheres representing $\gamma$ consists of a sum of lagrangian two-spheres

$$
\left(S^{2}\right)_{1} \cup \cdots \cup\left(S^{2}\right)_{k},
$$

that may have isolated singularities, as well as branch points. If each surface is a branched immersion then it can be shown [SW] that each surface is special lagrangian and, therefore, holomorphic for some complex structure on the hyperkähler line determined by $g$. However using techniques similar to those used in the previous problem it can be shown that there is an integral lagrangian homology class and a hyperkähler metric such that no minimizer of area among lagrangian two-spheres consists solely of branched immersions. In particular, there is a lagrangian two-sphere which is a minimizer of area among lagrangians that is not regular (i. e., is not a branched immersion). We will briefly describe this argument. A simpler proof of this result, using different techniques, has been given in [W]. Finally we give a new proof, along the lines of the arguments in [MW], of a theorem of Donaldson $[\mathrm{D}]$ relating stability, holomorphicity and the normal Euler number of a surface in a K3 surface. This result suggests that the class we use in

the construction of our main result is optimal in a certain sense. The work on this paper began at the IPAM workshop: The Geometry of Lagrangian Submanifolds held at IPAM in April, 2003. The authors are indebted to IPAM for the hospitality they extended to us during this workshop. The first author would also like to thank Mark Gross for many useful conversations about K3 surfaces.

\section{Preliminaries}

In this section we review basic results in Kähler geometry and the geometry of K3 surfaces that will be used in the proof of our result. For proofs see $[\mathrm{B}-\mathrm{P}-\mathrm{V}]$ and $[\mathrm{G}-\mathrm{H}]$.

Let $X$ be a K3 surface, that is, $X$ is a compact, complex, simply connected surface with trivial canonical bundle. Let

$$
L=-E_{8} \oplus-E_{8} \oplus H \oplus H \oplus H,
$$

define the intersection form on a vector space of real dimension 22 . Set $L_{\mathbb{C}}=L \otimes \mathbb{C}$ with the intersection form extended complex linearly. For any $\Omega \in L_{\mathbb{C}}$ we denote $[\Omega] \in \mathcal{P}\left(\mathcal{L}_{\mathbb{C}}\right)$ the corresponding line. It is known that $H^{2}(X, \mathbb{Z})$ is free of rank 22 and the intersection form on $H^{2}(X, \mathbb{Z})$ is given 
by $L$. In particular, $b_{+}^{2}=3$ and $b_{-}^{2}=19$. A marking of $X$ is a choice of basis,

$$
\left\{\alpha_{0}, \ldots, \alpha_{7}, \beta_{0}, \ldots, \beta_{7}, \xi_{1}, \xi_{2}, \xi_{3}, \eta_{1}, \eta_{2}, \eta_{3}\right\}
$$

of $H^{2}(X, \mathbb{Z})$ that induces the intersection form $L$. Equivalently a marking of $X$ is the choice of an isometry $\phi: H^{2}(X, \mathbb{Z}) \rightarrow L$. The period domain $\mathcal{D}$ of $X$ is the projectivization of the set:

$$
\left\{\Omega \in L_{\mathbb{C}}: \Omega \cdot \Omega=0, \Omega \cdot \bar{\Omega}>0\right\} .
$$

The complex dimension of $\mathcal{D}$ equals 20 . If $\Omega$ is a holomorphic $(2,0)$-form on $X$ then the identities $\Omega \cdot \Omega=0$ and $\Omega \cdot \bar{\Omega}>0$ show that a marking of $X$ determines a point $[\Omega] \in \mathcal{D}$, called the period point of $X$. The first main theorem we require is the weak Torelli theorem:

Theorem 2.1. Two K3 surfaces are isomorphic (as complex surfaces) if and only if there are markings for them such that the corresponding period points are the same.

The second main theorem we require is:

Theorem 2.2. All points of the period domain $\mathcal{D}$ occur as period points of marked K3 surfaces.

A class $\omega \in H^{1,1}(X, \mathbb{R})$ that can be represented by a Kähler form is called a Kähler class. Clearly a Kähler class satisfies $\omega \cdot \omega>0$ and $\omega \cdot \Omega=0$. Note that the set $\left\{x \in H^{1,1}(X, \mathbb{R}): x \cdot x>0\right\}$ consists of two disjoint connected cones and that the Kähler classes, if they exist, all belong to one of these two cones. This cone is called the positive cone. Additional conditions on the Kähler classes arise from the Picard lattice. Let $j: H^{2}(X, \mathbb{Z}) \rightarrow H^{2}(X, \mathbb{R})$ and define the Picard lattice $S_{X}=H^{1,1}(X, \mathbb{R}) \cap \operatorname{Im} j\left(H^{2}(X, \mathbb{Z})\right)$. An element $\sigma \in S_{X}$ is called divisorial if there exists a divisor $D$ whose associated line bundle has Chern class $\sigma$. Then $\sigma$ is called effective if, in addition, $D$ can be chosen effective. The Kähler cone is defined to be the convex subcone of the positive cone consisting of those classes that have positive inner product with any effective class in $S_{X}$. The Kähler cone contains all Kähler classes. When $X$ is a K3 surface the characterization of the Kähler cone becomes particularly simple. A nonsingular curve $\gamma$ in $X$ is called nodal if $\gamma \cdot \gamma=-2$.

Theorem 2.3. For a K3 surface the Kähler cone consists of the classes $\omega \in H^{1,1}(X, \mathbb{R})$ that satisfy: (i) $\omega \cdot \omega>0$, (ii) $\omega \cdot \Omega=0$ and (iii) $\omega \cdot \gamma>0$, for all nodal curves $\gamma$ in $X$.

It is a consequence of the surjectivity of the refined period map that every class in the Kähler cone is a Kähler class. Consequently, Yau's theorem on the existence of Kähler Ricci flat metrics on K3 surfaces can be stated as:

Theorem 2.4. Let $(X, \omega)$ be a K3 surface where $\omega \in H^{1,1}(X, \mathbb{R})$ lies in the Kähler cone. Then there is a unique hyperkähler metric on $X$ whose Kähler form represents the class $\omega$. 
If $X$ is a Kähler surface and $\Sigma$ is a possibly singular holomorphic curve of genus $g$ in $X$ the adjunction formula is:

$$
\Sigma \cdot \Sigma \geq c_{1}(X) \cdot \Sigma+2 g-2,
$$

with equality when $\Sigma$ is nonsingular. When $X$ is a K3 surface this becomes:

$$
\Sigma \cdot \Sigma \geq 2 g-2 \geq-2 .
$$

If $X$ is a K3 surface, we say a (singular) holomorphic curve $\Sigma$ is a (-2)curve if $\Sigma \cdot \Sigma=-2$ (equivalently, if its Poincaré dual $\alpha$ satisfies $\alpha \cdot \alpha=-2$ ). From the adjunction formula it follows that if $\Sigma$ is a $(-2)$-curve then $\Sigma$ is a nonsingular rational curve.

We conclude this section with some results on lagrangian stationary surfaces in Kähler-Einstein surfaces (see [S-W 1]). Let $N$ be a Kähler-Einstein surface and $\Sigma$ be a lagrangian submanifold. We say $\Sigma$ is lagrangian stationary if the volume is stationary for arbitrary smooth variations preserving the lagrangian constraint.

Theorem 2.5. A closed, branched immersed, lagrangian surface in a KählerEinstein surface is a classical minimal surface if and only if it is lagrangian stationary.

Consequently,

Corollary 2.6. A closed, branched immersed, lagrangian stationary surface in a K3 surface, with a hyperkähler metric $g$, is special lagrangian. In particular, a closed, branched immersed, lagrangian stationary surface $\Sigma$ is a J-holomorphic curve with respect to a complex structure $J$ on the hyperkähler line of $g$.

Note that these results require regularity of the lagrangian stationary submanifold.

\section{The Results}

In one of the $E_{8}$ 's in the intersection form of the K3 surface label the four classes $\alpha_{0}, \alpha_{1}, \alpha_{2}, \alpha_{3} \in H_{2}(X, \mathbb{Z})$ that satisfy: $\alpha_{0} \cdot \alpha_{i}=1, i=1,2,3$, $\alpha_{i} \cdot \alpha_{j}=0$ for $i \neq j, i, j=1,2,3, \alpha_{0} \cdot \alpha_{0}=-2$, and $\alpha_{i} \cdot \alpha_{i}=-2$.

Lemma 3.1. There is a complex structure $[\Omega]$ on the marked K3 surface $X$, determined by the complex 2-form $\Omega$, that satisfies:

(1) $\operatorname{span}_{\mathbb{Z}}\left\{\alpha_{0}, \alpha_{1}, \alpha_{2}, \alpha_{3}\right\} \subset H^{1,1}(X, \mathbb{C}) \cap H^{2}(X, \mathbb{Z})$.

(2) For each $t, 0<t<1$ there is a Kähler class $\omega_{t}$ in the Kähler cone determined by $\Omega$ such that $\omega_{t} \cdot \alpha_{0}=t, \omega_{t} \cdot \alpha_{i}=1$ for $i=1,2,3$ and otherwise $\omega_{t}$ is fixed in $t$.

(3) For all $t \in(0,1)$, there exists $\lambda_{t}>0$ such that $\omega_{t} \cdot \omega_{t}=\frac{1}{2} \Omega_{t} \cdot \overline{\Omega_{t}}$, where $\Omega_{t}:=\lambda_{t} \Omega$. Note that $\left[\Omega_{t}\right]=[\Omega]$, i. e., the complex structure determined by $\Omega_{t}$ is the same as that determined by $\Omega$. 
(4) $\sqrt{\left(\omega_{t} \cdot \gamma\right)^{2}+\left|\Omega_{t} \cdot \gamma\right|^{2}} \geq 1$, for all $\gamma \in H^{2}(X, \mathbb{Z})$ such that $\gamma \cdot \gamma \geq-2$ except when $\gamma= \pm \alpha_{0}$. Equality holds if and only if $\gamma= \pm \alpha_{i}$ for $i=1,2,3$.

Proof. Let a marking of $X$ be given by,

$$
\left\{\alpha_{0}, \ldots, \alpha_{7}, \beta_{0}, \ldots, \beta_{7}, \xi_{1}, \xi_{2}, \xi_{3}, \eta_{1}, \eta_{2}, \eta_{3}\right\},
$$

where the classes $\alpha_{0}, \alpha_{1}, \alpha_{2}, \alpha_{3}$ are as described above. Recall that $\xi_{i} \cdot \xi_{j}=0$, $\eta_{i} \cdot \eta_{j}=0$, for all $i, j$, that $\xi_{i} \cdot \eta_{j}=0$ for $i \neq j$ and that $\xi_{i} \cdot \eta_{i}=1$ for all $i$. Therefore, $\left(\xi_{i}-\eta_{i}\right) \cdot\left(\xi_{j}+\eta_{j}\right)=0$ for all $i, j$ and $\left(\xi_{i}-\eta_{i}\right)^{2}=-2,\left(\xi_{i}+\eta_{i}\right)^{2}=2$ for all $i$.

Define the period point $\Omega$ as follows:

$$
\begin{gathered}
\Omega \cdot \alpha_{k}=\Omega \cdot \beta_{k}=0, \quad \text { for } k=0, \ldots, 7, \\
\Omega \cdot\left(\xi_{j}-\eta_{j}\right)=0, \text { for } j=1,2,3, \\
\Omega \cdot\left(\xi_{j}+\eta_{j}\right)=\sigma_{j}+i \tau_{j}, \quad \text { for } j=1,2,3,
\end{gathered}
$$

where the vectors

$$
\sigma=\left(\sigma_{1}, \sigma_{2}, \sigma_{3}\right), \quad \tau=\left(\tau_{1}, \tau_{2}, \tau_{3}\right)
$$

satisfy $|\sigma|=|\tau|>0$ and $\sigma \cdot \tau=0$. Choose $\sigma$ so that no rational linear combination of its components vanishes. It follows that $\Omega$ satisfies $\Omega \cdot \Omega=0$ and $\Omega \cdot \bar{\Omega}>0$ and therefore, by the Torelli theorem, $\Omega$ defines a complex structure. By the choice of $\sigma$ no integral homology class containing a multiple of $\xi_{j}+\eta_{j}$ for $j=1,2,3$ can be represented by a holomorphic curve.

Define the Kähler class $\omega_{t}$ for $0<t<1$ as follows:

$$
\begin{gathered}
\omega_{t} \cdot \alpha_{0}=t, \omega_{t} \cdot \alpha_{j}=1, \text { for } j=1,2,3, \\
\omega_{t} \cdot \alpha_{j}=2, \quad \text { for } j=4, \ldots, 7, \\
\omega_{t} \cdot \beta_{j}=2, \quad \text { for } j=0, \ldots, 7, \\
\omega_{t} \cdot\left(\xi_{j}-\eta_{j}\right)=2, \quad \text { for } j=1,2,3, \\
\omega_{t} \cdot\left(\xi_{j}+\eta_{j}\right)=\rho_{j}, \text { for } j=1,2,3,
\end{gathered}
$$

where the vector

$$
\rho=\left(\rho_{1}, \rho_{2}, \rho_{3}\right)
$$

satisfies $\rho \cdot \sigma=\rho \cdot \tau=0$. By choosing $|\rho|$ sufficiently large we can ensure that $\omega_{t} \cdot \omega_{t}>0$. The classes of nodal curves are integral linear combinations of $\left\{\alpha_{0}, \ldots, \alpha_{7}, \beta_{0}, \ldots, \beta_{7},\left(\xi_{1}-\eta_{1}\right),\left(\xi_{2}-\eta_{2}\right),\left(\xi_{3}-\eta_{3}\right)\right\}$ with coefficients that are non-negative. Therefore $\omega_{t} \cdot \gamma>0$ for all nodal curves. It follows that $\omega_{t}$ lies in the Kähler cone determined by $\Omega$.

Item (3) is established simply by multiplying $\sigma$ and $\tau$ by an appropriate choice of $\lambda_{t}>0$.

For the fourth item, suppose that $\gamma \cdot \gamma \geq-2$ and $\sqrt{\left(\omega_{t} \cdot \gamma\right)^{2}+\left|\Omega_{t} \cdot \gamma\right|^{2}} \leq 1$. We will show that, by taking $|\rho|$ (and therefore $\left|\sigma_{t}\right|$ and $\left|\tau_{t}\right|$ ) sufficiently large, $\gamma$ then has to be one of $\pm \alpha_{0}, \pm \alpha_{1}, \pm \alpha_{2}, \pm \alpha_{3}$.

Decompose $\gamma$ into its self-dual and anti self-dual parts $\gamma_{+}$and $\gamma_{-}$. Thus $\gamma_{+}=\sum_{i=1}^{3} n_{i}\left(\xi_{i}+\eta_{i}\right), n_{i} \in \frac{1}{2} \mathbb{Z}$ and $\gamma_{-} \in \operatorname{span}_{\mathbb{Z}}\left\{\alpha_{0}, \ldots, \alpha_{7}, \beta_{0}, \ldots, \beta_{7}, \frac{1}{2}\left(\xi_{1}-\right.\right.$ 
$\left.\left.\eta_{1}\right), \frac{1}{2}\left(\xi_{2}-\eta_{2}\right), \frac{1}{2}\left(\xi_{3}-\eta_{3}\right)\right\}$. As a first step, we shall show that $\gamma_{+}$has to vanish when $|\rho|$ is sufficiently large. Let $\langle\cdot, \cdot\rangle$ and $|\cdot|$ denote the Euclidean inner product and norm on $\mathbb{R}^{3}$. Then, letting $n:=\left(n_{1}, n_{2}, n_{3}\right)$ we get:

$$
-2 \leq \gamma \cdot \gamma=2|n|^{2}+\left(\gamma_{-} \cdot \gamma_{-}\right)
$$

where

$$
|n|^{2}=\frac{\left|\left\langle n, \sigma_{t}\right\rangle\right|^{2}}{\left|\sigma_{t}\right|^{2}}+\frac{\left|\left\langle n, \tau_{t}\right\rangle\right|^{2}}{\left|\tau_{t}\right|^{2}}+\frac{|\langle n, \rho\rangle|^{2}}{|\rho|^{2}} .
$$

We need to show that $n=(0,0,0)$. If not, then $|n|^{2} \geq 1 / 4$. Furthermore, by assumption, $\left|\Omega_{t} \cdot \gamma\right| \leq 1$ and so, $\left|\left\langle n, \sigma_{t}\right\rangle\right| \leq 1 / 2$ and $\left|\left\langle n, \tau_{t}\right\rangle\right| \leq 1 / 2$. Putting these inequalities in (3.2) yields

$$
|\langle n, \rho\rangle|^{2} \geq|n|^{2}|\rho|^{2}\left(1-\frac{2}{\left|\sigma_{t}\right|^{2}}-\frac{2}{\left|\tau_{t}\right|^{2}}\right) .
$$

It will be useful to observe that $\left|\sigma_{t}\right|^{2}=\left|\tau_{t}\right|^{2}=\omega_{t} \cdot \omega_{t}$ for all $t \in(0,1)$ and that there exists $C>0$ such that for all $t \in(0,1)$,

$$
\frac{1}{2}|\rho|^{2} \geq \omega_{t} \cdot \omega_{t} \geq \frac{1}{2}|\rho|^{2}-C \text {. }
$$

It follows that, for sufficiently large $|\rho|$,

$$
|\langle n, \rho\rangle|^{2} \geq \frac{1}{2}|n|^{2}|\rho|^{2} .
$$

Now $\omega_{t} \cdot \gamma=2\langle n, \rho\rangle+\omega_{t} \cdot \gamma_{-}$. By our assumption $\left|\omega_{t} \cdot \gamma\right| \leq 1$. Therefore $\left|\omega_{t} \cdot \gamma_{-}\right| \geq 2|\langle n, \rho\rangle|-1$. From this we deduce

$$
\left(\omega_{t} \cdot \omega_{t}\right)\left(-\gamma_{-} \cdot \gamma_{-}\right) \geq\left(\omega_{t} \cdot \gamma_{-}\right)^{2} \geq(2|\langle n, \rho\rangle|-1)^{2} \geq 3\left(|\langle n, \rho\rangle|^{2}-1\right) .
$$

Substituting into (3.1) and making use of (3.4) and (3.3) yields:

$$
\left(|n|^{2}+1\right)|\rho|^{2} \geq 2\left(|n|^{2}+1\right)\left(\omega_{t} \cdot \omega_{t}\right) \geq\left(-\gamma_{-} \cdot \gamma_{-}\right)\left(\omega_{t} \cdot \omega_{t}\right) \geq \frac{3}{2}|n|^{2}|\rho|^{2}-3 .
$$

Clearly this cannot hold for arbitrarily large $|\rho|$ unless $n=(0,0,0)$.

Now that we have shown that $\gamma_{+}=0$, we see that $-2 \leq \gamma \cdot \gamma=\gamma_{-} \cdot \gamma_{-} \leq$ -2 . It follows that $\gamma \cdot \gamma=-2$ and that $\gamma \in \operatorname{span}_{\mathbb{Z}}\left\{\alpha_{0}, \ldots, \alpha_{7}\right\}$ or $\gamma \in$ $\operatorname{span}_{\mathbb{Z}}\left\{\beta_{0}, \ldots, \beta_{7}\right\}$. ( $\gamma$ cannot be one of $\pm\left(\xi_{i}-\eta_{i}\right), i=1,2,3$ because $\omega_{t} \cdot\left(\xi_{i}-\right.$ $\left.\eta_{i}\right)=2$ by construction and $\left|\omega_{t} \cdot \gamma\right| \leq 1$ by assumption.) Suppose that $\gamma \in$ $\operatorname{span}_{\mathbb{Z}}\left\{\alpha_{0}, \ldots, \alpha_{7}\right\}$. A tedious calculation shows that if $\gamma=\sum_{i=0}^{7} m_{i} \alpha_{i}, m_{i} \in$ $\mathbb{Z}$ and $\gamma \cdot \gamma=-2$ then $m_{0}, \ldots, m_{7}$ must all have the same sign. Actually, there is a theoretical reason for this. The set $\left\{\gamma \in \operatorname{span}_{\mathbb{Z}}\left\{\alpha_{0}, \ldots, \alpha_{7}\right\}: \gamma \cdot \gamma=\right.$ $-2\}$ is the root system of type $-E_{8}$; there are 240 such roots. (See, for example, $[\mathrm{H}]$, pages 472 and 473.) $\left\{\alpha_{0}, \ldots, \alpha_{7}\right\}$ is a basis of this root system and therefore, if $\gamma$ is a root, then $\gamma=\sum_{i=0}^{7} m_{i} \alpha_{i}, m_{i} \in \mathbb{Z}$ and $m_{0}, \ldots, n_{7}$ all have the same sign. The same applies if $\gamma \in \operatorname{span}_{\mathbb{Z}}\left\{\beta_{0}, \ldots, \beta_{7}\right\}$. Item (4) now follows easily.

Remark: The class $\alpha_{4}-\alpha_{5}$ shows that (4) does not hold if $\gamma \cdot \gamma \leq-4$. 
By Yau's theorem for each $0<t<1$ there is a unique hyperkähler metric $g_{t}$ with Kähler form in the class of $\omega_{t}$. Note that the complex structure $[\Omega]$ is fixed.

Using the Riemann-Roch theorem it follows that the indecomposable classes $\alpha_{0}, \alpha_{1}, \alpha_{2}, \alpha_{3}$ can be represented by embedded holomorphic -2 curves that we denote, respectively $S_{0}, S_{1}, S_{2}, S_{3}$, [B-P-V, VIII 3.6]. These curves do not change with $t$.

The sequence of Ricci flat Kähler metrics $\left\{g_{t}\right\}$ on $X$, as $t \rightarrow 0$, has been studied by R. Kobayashi $[\mathrm{K}]$. Denote the orbifold obtained from $X$ by blowing down $S_{0}$ by $X_{0}$. Then $X_{0}$ has one orbifold point $p$ and an orbifold Kähler Ricci flat metric $g_{0}$ which is singular only at $p$. (The existence of $g_{0}$ was established in $[\mathrm{KT}]$, Theorem 1, p.348.) Furthermore, according to Theorem 21 in $[\mathrm{K}]$, the metrics $g_{t}$ converge smoothly on compact subsets of $X \backslash S_{0}$ to the metric $g_{0}$ on $X_{0} \backslash\{p\}$.

Let $\mathcal{N}$ be a tubular neighborhood of $S_{0}$ in $X$ and let $\bar{S}_{i}:=\pi\left(S_{i} \cap \mathcal{N}\right)$ where $\pi: X \rightarrow X_{0}$ is the blow down projection. Of course, $\bar{S}_{1}, \bar{S}_{2}$ and $\bar{S}_{3}$ all meet at $p$ in $X_{0}$. In order to understand this intersection fully, we shall recall the explicit description of $\pi$. The tubular neighborhood $\mathcal{N}$ is biholomorphic to a neighborhood of the zero section of $T^{*} \mathbb{C P}^{1}$; we shall therefore identify this zero section with $S_{0}$. The blow down of $T^{*} \mathbb{C P}^{1}$ along $S_{0}$ is the quadratic cone $\mathbb{C}^{2} / \mathbb{Z}_{2}$, where $\mathbb{Z}_{2}$ acts on $\left(z_{1}, z_{2}\right)$ by $\left(z_{1}, z_{2}\right) \mapsto$ $\left(-z_{1},-z_{2}\right)$. Let $\sigma: \mathbb{C}^{2} \rightarrow \mathbb{C}^{2} / \mathbb{Z}^{2}$ be the natural projection. We shall exhibit a holomorphic double covering $\rho: \mathbb{C}^{2} \backslash\{(0,0)\} \rightarrow T^{*} \mathbb{C P}^{1} \backslash S_{0}$ such that $\pi \circ \rho=\sigma$. For this purpose, cover $T^{*} \mathbb{C P}^{1}$ by two coordinate charts $\mathcal{U} \cong$ $\mathcal{U}^{\prime} \cong \mathbb{C}^{2}$ with coordinates $(u, \xi),\left(u^{\prime}, \xi^{\prime}\right)$ respectively; here $u$ and $u^{\prime}$ denote Euclidean coordinates on the base $\mathbb{C P}^{1}$, and $\xi$ and $\xi^{\prime}$ parametrize the fibers of the bundle $T^{*} \mathbb{C P} \mathbb{P}^{1} \rightarrow \mathbb{C P}^{1}$. This means that

$$
\mathcal{U} \cap \mathcal{U}^{\prime}=\mathbb{C}^{*} \times \mathbb{C} \text { and, if }\left(u^{\prime}, \xi^{\prime}\right) \in \mathcal{U} \cap \mathcal{U}^{\prime} \text { then }\left(u^{\prime}, \xi^{\prime}\right) \sim\left(1 / u, u^{2} \xi\right) .
$$

Define $\rho: \mathbb{C}^{2} \backslash\{(0,0)\} \rightarrow T^{*} \mathbb{C P}^{1} \backslash S_{0}$ by

$$
\rho\left(z_{1}, z_{2}\right)= \begin{cases}\left(z_{1} / z_{2}, z_{2}^{2}\right) \in \mathcal{U} \backslash\left\{\mathcal{U} \cap S_{0}\right\}, & \text { if } z_{2} \neq 0, \\ \left(z_{2} / z_{1}, z_{1}^{2}\right) \in \mathcal{U}^{\prime} \backslash\left\{\mathcal{U}^{\prime} \cap S_{0}\right\}, & \text { if } z_{1} \neq 0 .\end{cases}
$$

Note that $\rho\left(z_{1}, z_{2}\right)=\rho\left(-z_{1},-z_{2}\right)$ and therefore, $\rho$ descends to a biholomorphic map $\bar{\rho}: \mathbb{C}^{2} \backslash\{(0,0)\} / \mathbb{Z}_{2} \rightarrow T^{*} \mathbb{C P}^{1} \backslash S_{0}$. It follows that $\pi(q)=\bar{\rho}^{-1}(q)$ if $q \notin S_{0}$ and $\pi(q)=[(0,0)]$ if $q \in S_{0}$; this establishes $T^{*} \mathbb{C P}^{1}$ as the minimal resolution of the cone $\mathbb{C}^{2} / Z_{2}$.

Let $\hat{S}_{i}:=\sigma^{-1}\left(\bar{S}_{i}\right) \subset \mathbb{C}^{2}$. We shall show that $\hat{S}_{1}, \hat{S}_{2}$ and $\hat{S}_{3}$ intersect pairwise transversally at $(0,0)$. Let $p_{i}:=S_{i} \cap S_{0}$. We may as well assume that $p_{i}=\left(u_{i}, 0\right) \in \mathcal{U}$. Since $S_{i}$ intersects $S_{0}$ transversely at $p_{i}$, there exist holomorphic functions $f_{i}: B_{\varepsilon} \rightarrow \mathbb{C}, B_{\varepsilon}:=\{w \in \mathbb{C}:|w|<\varepsilon\}$, such that $S_{i} \cap \mathcal{N}=\left\{\left(f_{i}(w), w\right): w \in B_{\varepsilon}\right\}$. It follows that $\hat{S}_{i}=\left\{\left(\zeta f_{i}\left(\zeta^{2}\right), \zeta\right):\left|\zeta^{2}\right|<\varepsilon\right\}$. But $f_{i}(0)=u_{i}$ and $u_{1}, u_{2}, u_{3}$ are all distinct. Therefore, $\hat{S}_{1}, \hat{S}_{2}$ and $\hat{S}_{3}$ are all nonsingular and intersect pairwise transversally at $(0,0)$. 
To say that $g_{0}$ is a Kähler Ricci flat orbifold metric on $X_{0}$ means that $\hat{g_{0}}:=\sigma^{*}\left(g_{0} \mid \mathcal{V}\right), \mathcal{V}:=\pi(\mathcal{N})$, is a smooth $\mathbb{Z}_{2}$ invariant metric on $\sigma^{-1}(\mathcal{V})$, which is a neighbourhood of $(0,0) \in \mathbb{C}^{2}$. Two of $\hat{S}_{1}, \hat{S}_{2}$ and $\hat{S}_{3}$ must intersect nonorthogonally with respect to $\hat{g_{0}}$. Renumbering we can suppose that this pair is $\hat{S}_{1}, \hat{S}_{2}$. Reverse the orientation on $\hat{S}_{2}$. Then the two tangent planes of $\hat{S}_{1}$ and $\hat{S}_{2}$ at $(0,0)$ intersect non-orthogonally at $(0,0)$ and define an orientation on $\mathbb{C}^{2}$ which is opposite to the canonical one. Hence they do not form an area minimizing configuration in $T_{(0,0)}(\mathcal{V})[\mathrm{L}]$, [Mo]. It follows that in $\mathcal{V}$, there are discs $D_{i} \subset \hat{S}_{i}$ centered at $(0,0) \in \hat{S}_{1} \cap \hat{S}_{2}$ and an annulus $A$ in $\mathcal{V}$ (a "Lawlor neck") with boundary $\partial A=\partial D_{1} \cup \partial D_{2}$ such that,

$$
\operatorname{area}_{\hat{g_{0}}}(A)<\operatorname{area}_{\hat{g_{0}}}\left(D_{1} \cup D_{2}\right) \text {. }
$$

Note that the annulus can be chosen so that $A$ is disjoint from $(0,0)$. Then for $\varepsilon>0$ sufficiently small it remains true that,

$$
\operatorname{area}_{\hat{g_{0}}}(A)<\operatorname{area}_{\hat{g_{0}}}\left(\left(D_{1} \cup D_{2}\right) \backslash\left(D_{1} \cup D_{2}\right) \cap B_{\varepsilon}\right) .
$$

Since $\sigma$ restricted to $\mathcal{V} \backslash B_{\varepsilon}$ is a local isometry, it follows that in $X_{0} \backslash\{p\}$ :

$$
\begin{aligned}
\text { area }_{g_{0}}(\sigma(A)) & <\operatorname{area}_{g_{0}}\left(\sigma\left(\left(D_{1} \cup D_{2}\right) \backslash\left(D_{1} \cup D_{2}\right) \cap B_{\varepsilon}\right)\right) \\
& <\operatorname{area}_{g_{0}}\left(\sigma\left(D_{1} \cup D_{2}\right)\right) .
\end{aligned}
$$

The annulus $\sigma(A)$ can then be used to glue $\bar{S}_{1} \backslash \sigma\left(D_{1}\right)$ to $-\left(\bar{S}_{2} \backslash \sigma\left(D_{2}\right)\right)$ forming a piecewise $C^{1}$ two-sphere $\bar{S} \subset X_{0} \backslash\{p\}$. Clearly $\bar{S}$ has $g_{0}$-area strictly less than area $g_{0}\left(\bar{S}_{1}\right)+$ area $_{g_{0}}\left(\bar{S}_{2}\right)=2$. Since the metrics $g_{t}$ converge in $C^{\infty}$ uniformly on compact subsets of $X_{0} \backslash\{p\}$ to $g_{0}$, for $t$ sufficiently small, the two-sphere $S:=\bar{\rho}(\bar{S}) \subset X \backslash S_{0}$ has $g_{t}$-area strictly less than 2 in $\left(X, g_{t}\right)$. In conclusion, there is a two-sphere $S$ in $\left(X, g_{t}\right)$ that represents $\alpha_{1}-\alpha_{2}$ in integral homology such that, for sufficiently small $t$, the $g_{t}$-area of $S$ is strictly less than 2 . Recall that the $g_{t}$-area of each of the holomorphic curves $S_{1}, S_{2}$ and $S_{3}$ is 1 and of the holomorphic curve $S_{0}$ is $t$.

We say a surface $\Sigma$ in the $\mathrm{K} 3$ surface $(X, g)$ is $\mathcal{J}$-holomorphic if it is Jholomorphic for some $J \in \mathcal{J}$ the hyperkähler line of $g$. We will need the following elementary lemma.

Lemma 3.2. Suppose that $\Sigma$ is a surface in the $K 3$ surface $\left(X, g_{t}\right)$ that is $\mathcal{J}$-holomorphic. Then

$$
\operatorname{area}_{g_{t}}(\Sigma)=\sqrt{\left(\omega_{t} \cdot \gamma\right)^{2}+\left|\Omega_{t} \cdot \gamma\right|^{2}}
$$

where $\gamma$ is the Poincaré dual of $\Sigma$.

Proof. Suppose that $\Sigma$ is holomorphic with respect to the complex structure $J$ compatible with $g_{t}$ and let $\omega$ be the Kähler form of $\left(J, g_{t}\right)$. Then

$$
\operatorname{area}_{g_{t}}(\Sigma)=\int_{\Sigma} \omega=\gamma \cdot \omega
$$


Let $\gamma_{+}$be the self-dual part of $\gamma$. Then $(\gamma \cdot \omega)^{2}=\left(\gamma_{+} \cdot \gamma_{+}\right)(\omega \cdot \omega)$. But

$$
\gamma_{+} \cdot \gamma_{+}=\frac{\left(\gamma \cdot \omega_{t}\right)^{2}}{\omega_{t} \cdot \omega_{t}}+\frac{2\left|\gamma \cdot \Omega_{t}\right|^{2}}{\Omega_{t} \cdot \overline{\Omega_{t}}}
$$

The result follows using $\omega_{t} \cdot \omega_{t}=\frac{1}{2} \Omega_{t} \cdot \overline{\Omega_{t}}$ and $\omega \cdot \omega=\omega_{t} \cdot \omega_{t}$.

The following theorem is our main result.

Theorem 3.3. When $t$ is sufficiently small, no area minimizer of $\alpha_{1}-\alpha_{2} \in$ $H_{2}(X, \mathbb{Z})$, for the hyperkähler metric $g_{t}$, is the sum of surfaces each of which is $\mathcal{J}$-holomorphic.

Proof. Suppose, by way of contradiction, that some area minimizer of $\alpha_{1}-\alpha_{2}$ is the sum of surfaces each of which is $\mathcal{J}$-holomorphic. Recall that we have constructed a two-sphere representing this class with area strictly less than 2. First suppose that the area minimizer has one component $C$. Then $C$ is a branched minimally immersed surface that represents $\alpha_{1}-\alpha_{2}$. Thus,

$$
C \cdot C=\left(\alpha_{1}-\alpha_{2}\right) \cdot\left(\alpha_{1}-\alpha_{2}\right)=-4 \text {. }
$$

$C$ cannot be holomorphic, for any complex structure, as this would contradict the adjunction formula.

Next suppose that the area minimizer has, at least, two component surfaces $B$ and $C$. Each one is, by assumption, holomorphic for some complex structure. Therefore, provided that neither surface represents $\pm \alpha_{0}$ in homology, by Lemmas 3.1 and 3.2,

$$
\operatorname{area}(B)+\operatorname{area}(C) \geq 2 \text {. }
$$

But the sum of the areas of all component surfaces is stricly less than 2 . Therefore, at least one of the surfaces represents either $\alpha_{0}$ or $-\alpha_{0}$ in homology. The sum of the remaining surfaces then represents either (i) $\alpha_{1}-\alpha_{0}-\alpha_{2}$ or (ii) $\alpha_{1}+\alpha_{0}-\alpha_{2}$. Note that a single holomorphic curve cannot represent $\alpha_{1}+k \alpha_{0}-\alpha_{2}$ for any $k \in \mathbb{Z}$ (since $\left(\alpha_{1}+k \alpha_{0}-\alpha_{2}\right)^{2}=-4-2 k^{2}$ ). It follows that in both cases the set of remaining surfaces contains at least two components neither of which represents $\pm \alpha_{0}$ in homology. The previous argument shows that this is impossible. The result follows.

The previous arguments can be adapted to the study of minimizers among lagrangian two-spheres as follows: Recall that for each $0<t<1$ there is a unique hyperkähler metric $g_{t}$ with Kähler form in the class of $\omega_{t}$. Then for each $0<t<1$ there is an $S^{1}$ of Kähler forms, compatible with $g_{t}$, such that the -2 -curves $S_{1}$ and $S_{2}$ (used above) are lagrangian with respect to each form in the family. For each $t$ choose such a Kähler form and denote it $w_{t}$. Choosing a subsequence as $t \rightarrow 0$, we can suppose that the sequence $\left\{w_{t}\right\}$ converges to the (orbifold) symplectic form $w_{0}$ on $X_{0}$ and that $\bar{S}_{1}$ and $\bar{S}_{2}$ are lagrangian for $w_{0}$. The oriented lagrangian surfaces $\bar{S}_{1}$ and $-\bar{S}_{2}$ can be glued using a Lawlor neck to construct a lagrangian two-sphere $\bar{S}$ in $X_{0} \backslash\{p\}$ - $p$ being the orbifold singularity of $X_{0}$ - with $g_{0}$-area equal to $2-\varepsilon$, for 
some $\varepsilon>0$. It follows that there is a $\delta>0$ such that for all $t<\delta$ the twosphere $S:=\bar{\rho}(\bar{S}) \subset X \backslash S_{0}$ has $g_{t}$-area strictly less than $2-\varepsilon / 2$ in $\left(X, g_{t}\right)$. Now on any fixed compact subset of $X \backslash S_{0} w_{t}$ converges smoothly to $w_{0}$. Therefore, for $\delta$ sufficiently small and $t<\delta, S$ is approximately lagrangian with respect to $w_{t}$. In particular, it can be deformed to a lagrangian twosphere without changing its $g_{t}$-area by more than $\varepsilon / 2$. We conclude that for some $t$ sufficiently small there is a lagrangian two-sphere with $g_{t}$-area less than 2. Next we minimize area among lagrangian two-spheres that represent $\alpha_{1}-\alpha_{2}$.

Theorem 3.4. There is some lagrangian class in $H_{2}(X ; \mathbb{Z})$ that has an area minimizer among lagrangian two-sphere (for the hyperkähler metric $g_{t}$, $t$ sufficiently small) that is not a branched immersion.

Proof. Suppose, by way of contradiction, that every lagrangian two-sphere that is a $g_{t}$-area minimizer is regular (i. e., is a branched immersion). Then by the results of [SW] every such lagrangian two-sphere is holomorphic for some complex structure on the hyperkähler line determined by $g_{t}$. Consider an area minimizing sequence of lagrangian two-spheres that represent $\alpha_{1}-\alpha_{2}$ and recall that we have constructed a lagrangian two-sphere representing this class with area strictly less than 2 . The argument in the proof of Theorem 3.3 leads to a contradiction.

Remark: It follows from Theorem 3.4 that, on lagrangian area minimizers in a K3 surface, singularities other than branch points can and do occur.

To put this result in context, recall the constrained variational theory developed in $[\mathrm{SW}]$. Consider a homology class in a Kähler surface that can be represented by the image of a lagrangian map of a compact surface (a lagrangian homology class) and minimize area among such maps. Then in $[\mathrm{SW}]$ it is shown that a lagrangian minimizer exists, that the map is Lipschitz

and is an immersion except at a finite number of isolated points that are either (i) branch points, or (ii) singular points with non-flat tangent cone. The tangent cones can be described precisely and it can be shown that there is a Maslov index associated to each tangent cone (and hence to each singular point). If the map is a minimizer this index is \pm 1 . The sum of these indices equals the pairing of the first Chern class of the Kähler surface with the homology class of the minimizer. Thus, when this pairing is non-zero, a lagrangian minimizer must have singular points. However if the Kähler surface is Kähler-Einstein then this pairing vanishes and it is possible that the minimizer is always regular. More precisely, one could speculate that on a minimizer a pair of singularities with indices 1 and -1 could be shown to "cancel". Theorem 3.4 shows that this is not the case.

\section{The Motivating Idea}

The main construction in this paper is motivated by the following observation. Suppose a K3 surface $X$ admits two -2 -curves $S_{1}$ and $S_{2}$ that 
intersect. Denote $\left[S_{1}\right]=\alpha_{1}$ and $\left[S_{2}\right]=\alpha_{2}$. Suppose there is a hyperkähler metric $g$ on $X$ with Kähler form $\omega$ such that $\omega\left(S_{1}\right)=\omega\left(S_{2}\right)=1$ and such that $S_{1}$ and $S_{2}$ intersect non-orthogonally with respect to $g$. Then after gluing in a "Lawlor neck" a representative of the class $\alpha_{1}-\alpha_{2}$ can be constructed with $g$-area less than 2. (In fact, this representative can be taken to be lagrangian for the Kähler form Re $\Omega$.) Suppose an area minimizing sequence among two-spheres representing $\alpha_{1}-\alpha_{2}$ converges without bubbling. Then, by the adjunction formula, the area minimizer cannot be holomorphic. The area minimizing sequence cannot bubble into two spheres representing $\alpha_{1}$ and $-\alpha_{2}$, respectively, because then the sum of the areas of the bubbles is 2. Other possible bubbling can be ruled out by appropriate choice of complex structure and metric on $X$, as was done in Section 3.

We did not use this construction because of the technical difficulty of finding a hyperkähler metric on $X$ such that two -2-curves intersect nonorthogonally. Our construction in Section 3 exploits the existence of two -2curves that intersect non-orthogonally in an orbifold limit of the hyperkähler metrics on $X$. That is, we find the necessary -2-curves on the boundary of the moduli space of Calabi-Yau metrics rather than in the interior.

\section{Stability and Holomorphicity}

In this section we give a new proof, based on ideas in [MW], of a theorem of Donaldson [D]. Donaldson's result implies that an immersed area minimizer in a K3 surface with normal Euler number greater than -4 must be $\mathcal{J}$ holomorphic. If the area minimizer produced in Theorem 3.3 is immersed and consists of one component (i.e., there is no bubbling) then Donaldson's result shows that this example is optimal.

Theorem 5.1. (Donaldson) Let $\Sigma$ be an oriented immersed minimal surface in a 4-manifold $X$ equipped with a hyperkähler metric $g$. If the Euler number $e(\nu)$ of the normal bundle $\nu$ of $\Sigma$ in $X$ satisfies $e(\nu) \geq-3$ and $\Sigma$ is not holomorphic with respect to a Kähler structure of $g$ then $\Sigma$ cannot be strictly stable.

Proof. We start by recalling some of the basic geometry of an immersion $F: \Sigma \rightarrow X$ of an oriented surface $\Sigma$ in an oriented 4-manifold $X$ equipped with a Riemannian metric $g$. For more detail, refer to [MW]. We shall adopt the following notation:

(i) $(x, y)$ will denote isothermal co-ordinates for the metric on $\Sigma$ induced by $F . z=x+i y$ is then a local complex co-ordinate on $\Sigma$.

(ii) $F^{*}(T X)=\xi \oplus \nu, \quad \xi=$ tangent bundle of $\Sigma, \nu=$ normal bundle.

(iii) $\quad \xi_{\mathbb{C}}:=\xi \otimes_{\mathbb{R}} \mathbb{C}, \quad \nu_{\mathbb{C}}:=\nu \otimes_{\mathbb{R}} \mathbb{C}$,

$\xi_{\mathbb{C}}=\xi^{1,0} \oplus \xi^{0,1}, \quad \xi^{1,0}\left(\xi^{0,1}\right)=+i(-i)$ eigenspace of the rotation $J_{\Sigma}$ in $\xi$ by $90^{\circ}$ anticlockwise. Similarly, $\nu_{\mathbb{C}}=\nu^{1,0} \oplus \nu^{0,1}$; rotation by $90^{\circ}$ anticlockwise in $\nu$ is denoted by $J_{\nu}$.

(iv) Superscript $T(\perp)$ will denote orthogonal projection onto $\xi(\nu)$. 
(v) $D: \Gamma\left(F^{*}(T X) \rightarrow \Gamma\left(T^{*}(\Sigma) \otimes F^{*}(T X)\right.\right.$ is the connection on the pullback of the tangent bundle of $X$ induced by the Levi-Civita connection of $g$. We shall also make use of $D^{\prime}:=d z \otimes D_{\partial / \partial z}$ and $D^{\prime \prime}:=d \bar{z} \otimes D_{\partial / \partial \bar{z}}$. This notation is slightly different from that in the Appendix in [MW] but it is more conventional.

(vi) $\left.D\right|_{\Gamma(\nu)}=\nabla+B ; \quad \nabla:=\left.D^{\perp}\right|_{\Gamma(\nu)}$ is a metric compatible connection on $\nu$ and $B$ is the shape operator. $B^{\prime}$ is naturally defined by $B^{\prime} s:=$ $\left(D^{\prime} s\right)^{\top} \forall s \in \Gamma(\nu) . B^{\prime \prime}, \nabla^{\prime}$ and $\nabla^{\prime \prime}$ are defined similarly.

(vii) $\left.D\right|_{\Gamma(\xi)}=D^{\top}-B^{*} ; \quad D^{\top}$ is the Levi-Civita connection on $\Sigma$ and $B^{*}$ is the second fundamental form of $F$; it is dual to the shape operator $B$. Of course, $B^{* \prime}$ is defined by $B^{* \prime} s:=-\left(D^{\prime} \sigma\right)^{\perp} \forall \sigma \in \Gamma(\xi)$. $B^{* \prime \prime}$ is defined similarly.

(viii) $F$ is minimal if, and only if, $\left.B^{* \prime \prime}\right|_{\xi^{1,0}} \equiv 0$, which is equivalent to $\pi_{\xi}^{0,1} \circ B^{\prime \prime} \equiv 0$.

We now assume that $g$ is hyperkähler and we let $I_{1}, I_{2}, I_{3}$ be complex structures on $X$ which are parallel with respect to the Levi-Civita connection of $g$ and which define a quaternionic structure on $T X$ (and therefore, $\left.F^{*}(T X)\right)$. Let $J:=J_{\Sigma} \oplus J_{\nu}$. Then $J=\sum_{j=1}^{3}\left(\cos \alpha_{j}\right) I_{j}, \sum_{j=1}^{3} \cos ^{2} \alpha_{j} \equiv 1$. $\alpha_{j}$ is called the Kähler angle of $\Sigma$ with respect to $I_{j}$. A straightforward calculation shows that, for each $j \in\{1,2,3\}, I_{j}$ preserves $\Gamma\left(\nu^{1,0} \oplus \xi^{0,1}\right)$. This is essentially due to the fact that $\operatorname{Hom}\left(\nu^{1,0}, \xi^{0,1}\right)$ is the tangent space of the twistor space of $F^{*}(T X)$ at $J$. Let $\eta:=\nu^{1,0} \oplus \xi^{0,1}$ and let $\pi_{\eta}=\pi_{\nu}^{1,0} \oplus \pi_{\xi}^{0,1}$. Define a connection $D_{\eta}$ on $\Gamma(\eta)$ by $D_{\eta}:=\left.\pi_{\eta} \circ D\right|_{\Gamma(\eta)}$. Endow $\eta$ with a holomorphic structure by declaring $v \in \Gamma(\eta)$ to be holomorphic if, and only if, $D_{\eta}^{\prime \prime} v \equiv 0$ where $D_{\eta}^{\prime \prime}:=\left.\pi_{\eta} \circ D^{\prime \prime}\right|_{\Gamma(\eta)} . \quad \nu^{1,0}$ is endowed with the holomorphic structure defined by means of $\nabla^{\prime \prime}$ and $\xi^{0,1}$ is endowed with the holomorphic structure defined by means of $D^{\prime \prime \top}$. Assume that $F$ is minimal and write $v=s+\sigma, v \in \Gamma(\eta), s \in \Gamma\left(\nu^{1,0}\right), \sigma \in \Gamma\left(\xi^{0,1}\right)$. Then $D_{\eta}^{\prime \prime} v=\nabla^{\prime \prime} s-B^{* \prime \prime} \sigma+D^{\prime \prime \top} \sigma$ where we have used $\pi_{\xi}^{0,1} \circ B^{\prime \prime} \equiv 0$ by the minimality of $F$. Thus,

$$
v \in H^{0}(\eta) \Leftrightarrow \sigma \in H^{0}\left(\xi^{0,1}\right) \text { and } \quad \nabla^{\prime \prime} s=B^{* \prime \prime} \sigma
$$

where $s=\pi_{\nu}^{1,0} v$ and $\sigma=\pi_{\xi}^{0,1} v$. The proof of the theorem makes use of the second variation of area formula applied to sections $s=\pi_{\nu}^{1,0} v, v \in H^{0}(\eta)$. In $[\mathrm{MW}]$, the second variation of area formula was applied to holomorphic sections of $\nu^{1,0}$, which can also be viewed as holomorphic sections of $\eta$ (with $\sigma=0)$.

Let $\mathcal{R}$ denote the curvature operator of $X$ and let $e_{1}-i e_{2}$ be a local unitary section of $\xi^{1,0}$. Then, according to (A.10) in [MW], the second variation $\left(\delta^{2} A\right)(s)$ of area in the direction of $s \in \Gamma\left(\nu_{\mathbb{C}}\right)$ of a minimal surface $\Sigma$ in $X$ is given by:

$$
\left(\delta^{2} A\right)(s)=2 \int_{\Sigma}\left\{\left|\nabla^{\prime \prime} s\right|^{2}-\left|B^{\prime} s\right|^{2}-\frac{1}{2}\left\langle\mathcal{R} s \wedge\left(e_{1}-i e_{2}\right), s \wedge\left(e_{1}-i e_{2}\right)\right\rangle\right\} d A .
$$


If $s \in \Gamma\left(\nu^{1,0}\right)$ and $X$ is Kähler with zero scalar curvature then, as can be seen from Proposition 2.2 in [MW], the curvature term in the formula for $\left(\delta^{2} A\right)(s)$ drops out. Furthermore,

$$
\left|B^{\prime} s\right|^{2}=-\frac{1}{2}\left(K_{\xi}+K_{\nu}\right)|s|^{2}
$$

where $K_{\xi}$ is the Gauss curvature of $\Sigma$ and $K_{\nu}$ is the curvature of the connection $\nabla$ on $\nu$. This can be seen as follows: let $s=f\left(e_{3}-i e_{4}\right)$ where $e_{3}-i e_{4}$ is a local unitary section of $\nu^{1,0}$. Then

$$
B^{\prime} s=\frac{1}{2} f\left(h_{311}-h_{412}-i h_{312}-i h_{411}\right)\left(e_{1}+i e_{2}\right) \otimes\left(\theta_{1}+i \theta_{2}\right)
$$

where $\theta_{1}+i \theta_{2}$ is the local unitary section of $\left(\xi^{1,0}\right)^{*}$ dual to $e_{1}-i e_{2}$. Therefore,

$$
\left|B^{\prime} s\right|^{2}=\frac{1}{2}|s|^{2}\left(\left(h_{311}-h_{412}\right)^{2}+\left(h_{312}+h_{411}\right)^{2}\right) .
$$

Let $R_{A B C D}:=\left\langle\mathcal{R}\left(e_{A} \wedge e_{B}\right), e_{C} \wedge e_{D}\right\rangle$. Then, by the Gauss equation and the minimality of $F$,

$$
K_{\xi}=R_{1212}-\left(h_{311}^{2}+h_{312}^{2}+h_{411}^{2}+h_{412}^{2}\right)
$$

and, by the Ricci equation,

$$
K_{\nu}=R_{3412}+2\left(h_{311} h_{412}-h_{312} h_{411}\right) .
$$

Equation (5.1) now follows easily on noting that $R_{3412}+R_{1212}=\left\langle\mathcal{R}\left(e_{1} \wedge\right.\right.$ $\left.\left.e_{2}+e_{3} \wedge e_{4}\right), e_{1} \wedge e_{2}\right\rangle$ which, according to Proposition 2.2 in [M-W], is equal to zero for a Kähler surface $X$ with zero scalar curvature.

We are now in a position to prove Theorem 5.1. Let $v$ be a nontrivial holomorphic section of $\eta$ and let $s_{j}:=\pi_{\nu}^{1,0}\left(I_{j} v\right), j \in\{0,1,2,3\}$ where, for convenience, $I_{0}$ denotes the identity transformation on $\Gamma(\eta)$. Then, since for each $j, I_{j} v$ is a holomorphic section of $\eta$, we have $\nabla^{\prime \prime} s_{j}=B^{* \prime \prime} \sigma_{j}$ where $\sigma_{j}:=\pi_{\xi}^{0,1}\left(I_{j} v\right)$. Now by a calculation similar to that just carried out for the establishment of equation (5.1) one can show that

$$
\left|B^{* \prime \prime} \sigma_{j}\right|^{2}=-\frac{1}{2}\left(K_{\xi}+K_{\nu}\right)\left|\sigma_{j}\right|^{2}
$$

It follows that

$$
\sum_{j=0}^{3}\left(\delta^{2} A\right)\left(s_{j}\right)=\int_{\Sigma}\left(K_{\xi}+K_{\nu}\right) \sum_{j=0}^{3}\left(\left|s_{j}\right|^{2}-\left|\sigma_{j}\right|^{2}\right) d A .
$$

It is easy to see that

$$
\sum_{j=0}^{3}\left|s_{j}\right|^{2}=\sum_{j=0}^{3}\left|\sigma_{j}\right|^{2}=2|v|^{2} .
$$

Therefore, $\sum_{j=0}^{3}\left(\delta^{2} A\right)\left(s_{j}\right)=0$. But there are at least two values of $j$ (which we may as well assume to be 0 and 1$)$ for which $s_{j}$ does not vanish identically. Strict stability would then imply $\left(\delta^{2} A\right)\left(s_{j}\right)>0, j \in\{0,1\}$ and $\left(\delta^{2} A\right)\left(s_{k}\right) \geq 0, k \in\{2,3\}$. Hence strict stability and the existence of a nontrivial holomorphic section of $\eta$ lead to a contradiction. 
The proof of the theorem is completed by showing that, if $e(\nu) \geq-3$ and $F$ is not holomorphic with respect to any of $I_{1}, I_{2}$ and $I_{3}$, then $h^{0}(\eta)>0$. But by Riemann-Roch,

$$
h^{0}(\eta)=e(\nu)-e(\xi)+2-2 g+h^{0}\left(\left(\nu^{0,1} \oplus \xi^{1,0}\right) \otimes \kappa\right)
$$

where $\kappa$ is the canonical bundle of $\Sigma$. Now $\xi^{1,0} \otimes \kappa$ is the trivial line bundle and therefore, the last term is equal to $1+h^{0}\left(\nu^{0,1} \otimes \kappa\right)$. The result follows on noting that $e(\xi)=2 g-2$ and that, for each $j \in\{1,2,3\}, \pi_{\nu}^{0,1}\left(I_{j} F_{*}(\partial / \partial z)\right) \otimes$ $d z$ is a holomorphic section of $\nu^{0,1} \otimes \kappa$ which is nontrivial if $F$ is not holomorphic with respect to $I_{j}$.

\section{REFERENCES}

[AN] Arezzo, C. and La Nave, G., Minimal two spheres and Kähler-Einstein metrics on Fano manifolds, preprint available from www.math.unipr.it/ rivista/membri/AREZZO/Arezzo.html

[B-P-V] Barth, W., Peters, C. and Van de Ven, A. , Compact Complex Surfaces, SpringerVerlag, Berlin 1984.

[D] Donaldson, S. K., Moment maps and diffeomorphisms, Surv. Differ. Geom., VII, (2000), Int. Press, Somerville, MA, 107-127.

[G-H] Griffiths, P. and Harris, J., Principles of Algebraic Geometry, John Wiley and Sons, New York 1978.

[H] Helgason, S., Differential Geometry, Lie Groups, and Symmetric Spaces, Academic Press, 1978.

[K] Kobayashi, R., Moduli of Einstein Metrics on a K3 surface and Degeneration of Type I, Adv. Studies in Pure Math. 18-II (1990) 257-311.

[KT] Kobayashi, R. and Todorov, N., Polarized period map for generalized K3 surfaces and the moduli of Einstein metrics, Tohoku Math. J. 39 (1987) 341-363.

[L] Lawlor, G., The angle criterion, Invent. Math. 95 (1989) 437-446.

[M] Micallef, M., Stable minimal surfaces in Euclidean space, J. Differential Geom. 19 (1984), no. 1, 57-84.

[Mo] Morgan, F., On the singular structure of two-dimensional area minimizing surfaces in $\mathbb{R}^{n}$, Math. Ann. 261 (1982) 101-110.

[MW] Micallef, M. and Wolfson, J., The Second Variation of Area of Minimal Surfaces in Four-Manifolds, Math. Ann. 295 (1993) 245-267.

[SW] Schoen, R. and Wolfson, J., Minimizing areaamong lagrangian surfaces: the mapping problem, J. Diff. Geom. 58 (2001) 1-86.

[W] Wolfson, J., Lagrangian homology classes without regular minimizers, preprint. 